\title{
A Faster Genetic Algorithm for Substation Location and Network Design of Power Distribution Systems
}

\author{
Cristiane G. Tarôco \\ Programa de Pós Graduação em \\ Engenharia Elétrica \\ Universidade Federal de Minas Gerais \\ Av. Antônio Carlos, 6627 \\ Belo Horizonte, MG, Brazil \\ Email: cristiane@cpdee.ufmg.br
}

\author{
Eduardo G. Carrano \\ Dep. Electrical Engeneering \\ Universidade Federal de Minas Gerais \\ Av. Antônio Carlos, 6627 \\ Belo Horizonte, MG, Brazil \\ Email: egcarrano@ufmg.br \\ Ricardo H. C. Takahashi \\ Dep. Mathematics \\ Universidade Federal de Minas Gerais \\ Av. Antônio Carlos, 6627 \\ Belo Horizonte, MG, Brazil \\ Email: taka@mat.ufmg.br
}

\author{
Oriane M. Neto \\ Dep. Electrical Engeneering \\ Universidade Federal de Minas Gerais \\ Av. Antônio Carlos, 6627 \\ Belo Horizonte, MG, Brazil \\ Email: oriane@cpdee.ufmg.br
}

\begin{abstract}
In this paper, a genetic algorithm is employed to plan the medium and long term expansion of electric power distribution systems. The expansion planning task is modeled as a single-objective optimization problem in which the objective function is the monetary cost of the network. A new procedure is proposed to perform substation location jointly with network topology design. Such a procedure is performed during function evaluation, and it requires low computational cost. Results for a real eight bus energy system are presented. These results show that reasonable solutions can be reached in a computational time considerably lower than the one required by former methods.
\end{abstract}

\section{INTRODUCTION}

The electric distribution systems are usually spread over large geographical areas, what makes them the major responsible for energy losses and failures [1]. This means that the employment of optimization techniques in the design of such systems can lead to large economic savings. The loads connected to the energy system tend to grow along its operation time. As a consequence, electrical distribution systems have to be continuously re-designed and expanded [2]. The system expansion can be interpreted as two sub-problems:

- Substation location: sometimes it is necessary to install new substations in order to meet the load demands. The problem of substation location may be expressed in terms of continuous variables when the decision variables involved are the substation coordinates $(x$ and $y$ ), in problem instances in which a continuous variation of such coordinates is allowed. If the substation location changes, the conductors that connect it to the other network nodes have their lengths also changed, what can affect the optimal solution to be delivered [2].

- Network topology: the topology of the network (structure which connects the loads and the substations) must be also designed. It is necessary to define which pair of nodes are going to be directly connected and the type of conductor to be employed in the connection. This is a combinatorial problem and, due to the dependence of the solution with the system power flow, the objective function is non-linear and computationally expensive to be evaluated.

The optimization methods that can be used to solve the electric energy expansion problem have some limitations. Techniques like Branch-and-Bound [3] require exponential computational time, what can render their employment infeasible even for moderate instances. Discrete versions of Simplex [3] can be used to solve network design problems, but the non-linear objective function needs to be approximated to a first order approximation function -, what may compromise the final result precision.

The Evolutionary Algorithms (EAs) are a good choice to deal with network problem as they do not depend on mathematical premises like linearity or convexity. Due to their flexibility, the EAs have become prominent instruments to solve problems with mixed variables [4]. In the problem treated in this paper, the variables are mixed, since the substation position is represented by continuous variables and the network topology is represented by discrete variables.

Some works that used Evolutionary Algorithms to solve problems involving distribution energy systems can be mentioned:

- Genetic algorithms [5]: in [2] and [6] two genetic algorithms have been combined with a quasi-Newton BFGS algorithm for planning substation location and network design of power distribution systems.

- NSGA [7], NSGA-II [8] and SPEA [9]: these algorithms 
have been used for solving multiobjective instances of the problem in [10], [11] and [12].

- Particle Swarm Optimization (PSO) [13]: single-objective and multiobjective instances of the distribution network design problem have been handled by PSO algorithms in [14].

In this paper the distribution network expansion problem is solved using a genetic algorithm. This algorithm was chosen because there are several reports in literature of successful applications of such an algorithm in network optimization problems. The proposed algorithm tries to find the adequate places for installing substations and the optimal topology associated to these locations. When compared to [2] and [6], this new approach has the advantage of performing substation location inside the genetic algorithm, instead of using a separate optimization algorithm (quasi-Newton BFGS). It provides better treatment of problem coupling and it reduces considerably the time required for reaching the solution. In the instances considered here, this time has been reduced by a factor of about 10 , for a similar instance in the same simulation machine. The objective function considered here has been the total cost of the network. This cost comprises the investment required for installing the system and the amount of money that is spent every year with system maintenance and losses.

The paper is structured as follows:

- in section II, the problem formulation and the proposed methodology are discussed;

- the real expansion problem considered in this paper is presented in section III;

- in section IV, the results obtained for the problem are presented.

\section{Methodology}

\section{A. The problem formulation}

Three main aspects should be taken into account in power distribution system design:

- minimization of installation and expansion costs;

- minimization of maintenance costs along the operation time;

- minimization of costs related to generated but not delivered energy (losses) along the operation time.

These three aspects can be aggregated into a single monetary value by considering the present values of the second and third indicators [15], [11]. This function is shown in (1).

$$
\begin{aligned}
f(N, S)= & \sum_{i \in \mathcal{M}} Y[n(i)]\left\{B C[n(i)] \cdot l_{i}+\right. \\
& \left.\sum_{j=1}^{a t}\left\{M C[n(i)] \cdot l_{i}+L C_{i}\right\}(1-i r)^{j-1}\right\}+ \\
& \sum_{k \in \mathcal{S}} Y[n(k)]\left\{B C[n(k)] \cdot l_{k}[s(k)]+\right. \\
& \left.\sum_{l=1}^{a t}\left\{M C[n(k)] \cdot l_{k}[s(k)]+L C_{k}[s(k)]\right\}(1-i r)^{l-1}\right\}
\end{aligned}
$$

in which:

$f(N, S)$ is the monetary cost of the network;
$N$ is a vector of decision variables that represents the connections which are enabled in the network. If $n_{i}=p \neq 0$, then the connection $i$ is enabled and it is connected by a conductor of type $p$. If $n_{i}=0$, then the connection is disabled. The dimension of this vector is $1 \times(|\mathcal{M}|+|\mathcal{S}|)$;

$S$ is a vector of decision variables which represent the coordinates of the substations to be located;

$\mathcal{M}$ is the set of possible connections that do not involve the substations to be located;

$\mathcal{S}$ is the set of possible connections that involve at least one substation to be located;

at is the time horizon considered for cost computation in the design;

$n(i)$ is the edge involved in the $i$-th element of sets $\mathcal{M}$ or $\mathcal{S}$. It can assume value 0 (disabled), 1 (enabled with conductor type 1$), \ldots, p$ (enabled with conductor type $p$ );

$s(i)$ are the coordinates of the substation involved in the $i$-th element of the set $\mathcal{S}$. It is a pair $\left(x_{s(i)}, y_{s(i)}\right)$;

$Y[n(i)]$ is a function which assumes 1 if $n(i) \neq 0$ or 0 if $n(i)=0$;

$B C[n(i)]$ is the branch cost, in $\$ / k m$, of the conductor type $n(i)$;

$l_{i}$ is the length, in $k m$, of the edge $i$;

$M C[n(i)]$ is the maintenance cost, in $\$ / k m / y e a r$, of the conductor type $n(i)$;

$L C_{i}=8760 \cdot l^{f} \cdot e n^{t} \cdot P_{i}^{L}$ is the cost of the energy loss in edge $i$;

$i r$ is the interest rate;

$l_{k}[s(k)]$ is the length, in $k m$, of the edge $k$, as a function of the substation located in $s(k)$, whose position can be changed during design;

$L C_{k}[s(k)]$ is the cost of the energy loss in edge $k$, as a function of the substation located in $s(k)$, whose position can be changed during design;

$l^{f}$ is the loss factor;

$e n^{t}$ is the energy tax, in $\$ / k W h$;

$P_{i}^{L}$ is the energy loss in edge $i$, in $k W$.

Based on this function, it is possible to note that two sets of decision variables are involved, the network edges $(N)$ and the substation coordinates $(S)$.

There are some constraints that must be complied by the candidate solutions [16]:

- $c_{1}$ : all consumers must be served;

- $c_{2}$ : the distribution network must be radial (tree structure);

- $c_{3}$ : the current in each line cannot exceed the capacity of the conductor type;

- $c_{4}$ : the voltage in each load bus must be between the limits specified by regulatory rules (in Brazil it must be between $0.92 \mathrm{pu}$ and $1.08 \mathrm{pu}$ ).

Besides, there are other two constraints which must be considered for substation location:

- $c_{5}$ : the coordinates $x$ and $y$ have minimum and maximum boundaries;

- $c_{6}$ : sometimes, the substations cannot be installed in 
some regions, due to geographical accidents or technical reasons.

The algorithms proposed in this work intend to minimize the function in Eq.(1) in order to look for optimal distribution systems. These algorithms are described along the next sections.

\section{B. Proposed methodology}

In the proposed approach, a genetic algorithm has been employed to look for the best topology of the distribution system. This algorithm is the same proposed in [17], which is intended to perform tree network design using discrete extensions of continuous space properties. Each individual is represented by a vector and each position of the vector can assume value 0 (the edge is not enabled) or a positive integer which indicates the conductor type employed.

A new procedure has been proposed for placing the substations into the regions they can be installed. It works as follows:

1) given a resolution parameter $^{1} r$, split each region in which it is possible to install substations into discrete sets of equally spaced points (Fig. 1). Each of these points is a candidate position for the substations.

2) for each substation to be located:

a) set the initial position of the substation as the arithmetic mean of the coordinates of the vertices which are connected to it;

b) amongst the set of candidate positions, choose the one which is nearest to the position found in the previous step (Fig. 2).

3) evaluate $\mathrm{AC}$ power flow;

4) if necessary, fix the branch types in order to comply with constraint $c_{3}$

5) for each substation to be located:

a) set the new position of the substation as the weighed mean of the coordinates of the vertices which are connected to it. The weight of each node is the amount of active power which flows in the edge which connects it to the substation;

b) amongst the set of candidate positions, choose the one which is the nearest to the position found in the previous step.

6) update $\mathrm{AC}$ power flow.

This procedure is also executed inside the function evaluation block, in the highest level of the algorithm. This means that it is not necessary to perform any change on the algorithm structure. Differently from the previous approaches [2], the proposed one is able to handle with disjoint regions for installing the substations. It should be kept in mind that the parameter $r$ is strictly related with the number of candidate locations which are to be considered. By consequence, the quality of the solution delivered is also affected by $r$.

\footnotetext{
${ }^{1}$ This parameter defines the horizontal and vertical distances between the candidate SS positions.
}

The disjointly feasible regions for substation location presented in Fig. 1 can occur when geographical accidents are present in some parts of the region considered or due to technical reasons. Fig. 3 shows an example of a region where a substation must be installed and, due to geographical accidents, the feasible region is a disjoint set. The optimization algorithm proposed here can handle with such situations.

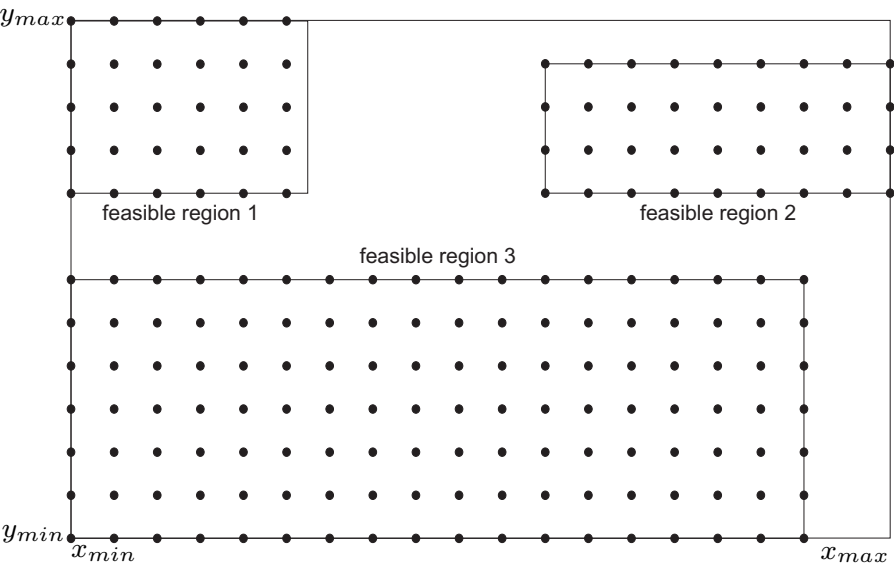

Fig. 1. Three disjoint feasible regions and their candidate positions.

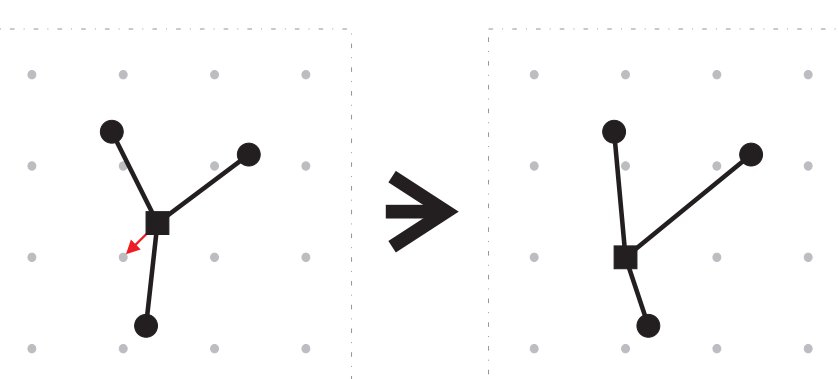

Fig. 2. Choosing the nearest candidate position for installing the substation.

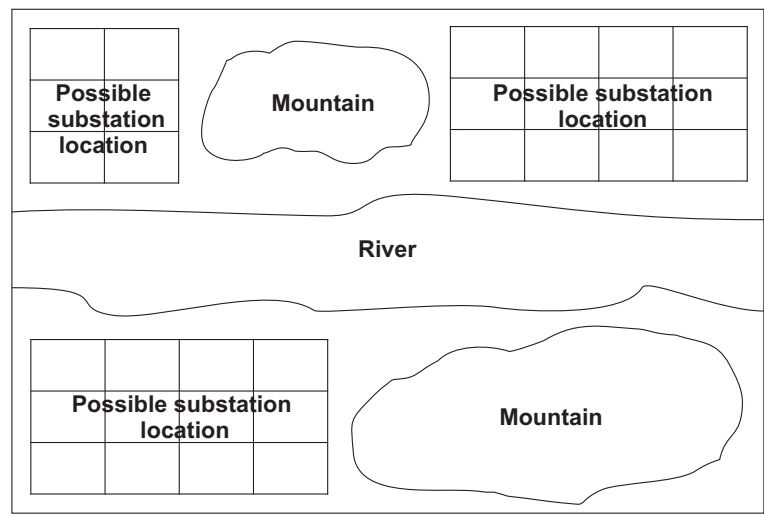

Fig. 3. Example of possible regions for substation location when geographical accidents are presented.

With regard to constraint handling:

- the algorithm inherently ensures that the constraints $c_{1}$, $c_{2}, c_{5}$ and $c_{6}$ are complied; 
- the constraint $c_{3}$ is handled by a correction mechanism that works as follows: $(i)$ after running the power flow, it is tested if the current in each branch is lower than its maximum capacity; ( $i i .1)$ if all branches meet the requirements, than nothing is changed; $(i i .2)$ otherwise, the branches which do not support the current are replaced by higher capacity ones. This is the fix mechanism cited in step 4 of the substation location procedure.

- the constraint $c_{4}$ is handled by a simple linear penalty function.

\section{TEST INSTANCE}

The proposed methodology has been used to solve a real expansion problem confronted by the Electric Energy Utility of Minas Gerais (CEMIG). The system has eight nodes and it is shown in Fig. 4. This area, which is situated in the north of Minas Gerais state, Brazil, is expanding its economic activity and its population. A new substation should be installed in 2005 , because the existing electric system facilities were no longer able to supply the energy demands. This led to the redesign of the network topology. This system is the same one considered in [2].

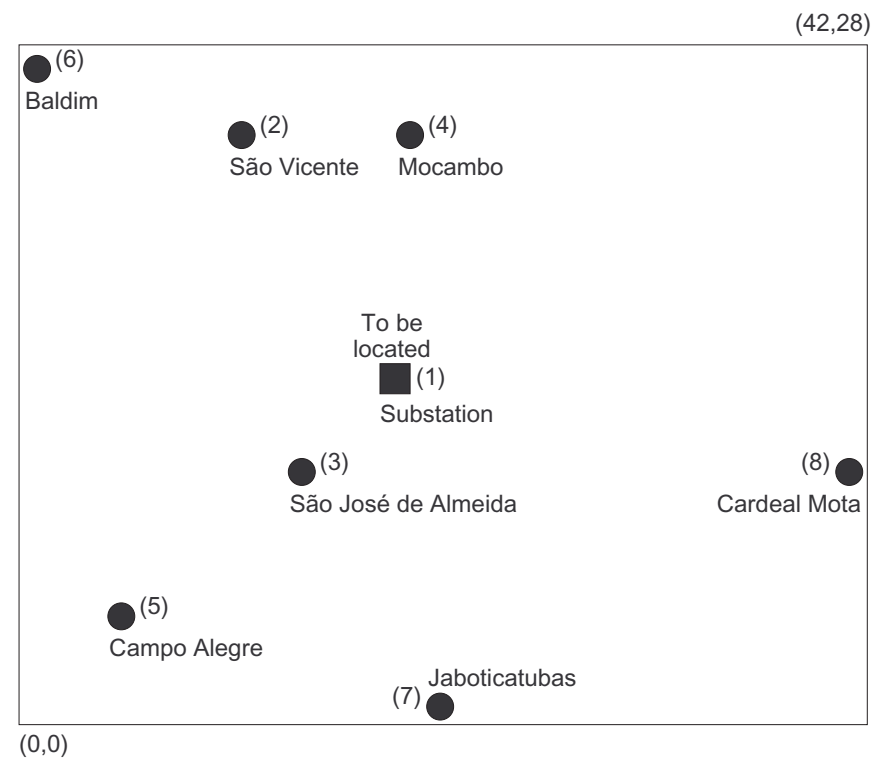

(6112-7840) UTM

Fig. 4. The eight bus system.

In this system, the substation can be placed in any place inside the rectangle $[(00,00) ;(42,28)]$. Besides, two types of conductors have been considered in design, as shown in Tab. I.

\section{RESULTS}

Initially the optimization algorithm has been performed for different future time horizons (from one to ten years), considering a resolution parameter $r=0.25 \mathrm{~km}$. The same loads have been considered for all the time horizons and the increase observed for objective function values along time is caused by the loss and maintenance costs, which are computed
TABLE I

CONDUCTOR CHARACTERISTICS.

\begin{tabular}{|c|c|c|c|c|c|c|}
\hline $\mathrm{A}$ & $\mathrm{B}$ & $\mathrm{C}$ & $\mathrm{D}$ & $\mathrm{E}$ & $\mathrm{F}$ & $\mathrm{G}$ \\
\hline 1 & 50 & 208 & 0.7394 & 0.2682 & 35000.00 & 975.00 \\
2 & 150 & 415 & 0.2469 & 0.2417 & 50000.00 & 975.00 \\
\hline
\end{tabular}

A - Conductor type;

B - Diameter $\left(\mathrm{mm}^{2}\right)$;

C - Nominal current $(A)$;

D - Resistance $(\Omega)$;

E - Reactance $(\Omega)$;

$\mathrm{F}$ - Installation cost $(\$ / \mathrm{km})$;

$\mathrm{G}$ - Maintenance cost $(\$ / \mathrm{km} /$ year $)$.

per year. Two different topologies have been obtained in the 10 simulations. They are shown in figures 5 and 6 .

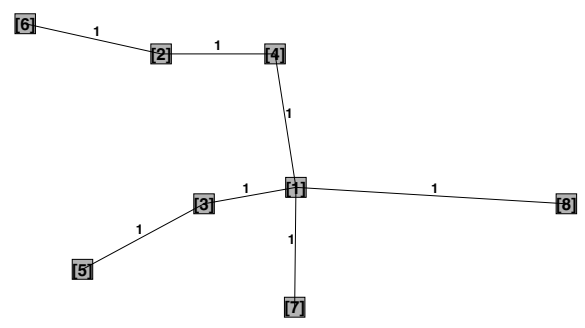

Fig. 5. Topology 1.

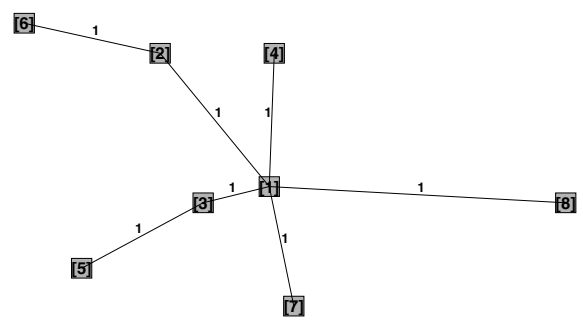

Fig. 6. Topology 2.

Tab. II shows the substation location, the topology of the network and the objective function value for the time horizons considered. Additionally, such an evolution of cost along time can be seen in Fig. 7. The change in the topology as larger time horizons are considered is due to the changes that occur in the energy losses annually. For shorter design horizons, the algorithm finds solutions with lower installation costs, since these costs are considerably higher than the amount spent with loss and maintenance. However, for longer time horizons, it is 
interesting to adopt topologies with lower losses, since those costs become significant in the total cost of the network.

TABLE II

NETWORK INFORMATION FOR EACH YEAR.

\begin{tabular}{|c|c|c|c|c|}
\hline Time horizon (years) & $x_{s(i)}$ & $y_{s(i)}$ & $f(N, S) \times 10^{6}$ & Topology \\
\hline 1 & 21 & 11.75 & 3.2540 & 1 \\
\hline 2 & 21 & 11.75 & 3.4870 & 1 \\
\hline 3 & 21 & 11.75 & 3.6966 & 1 \\
\hline 4 & 21 & 11.75 & 3.8853 & 1 \\
\hline 5 & 21 & 11.75 & 4.0551 & 1 \\
\hline 6 & 21 & 11.75 & 4.2079 & 1 \\
\hline 7 & 19 & 11.75 & 4.3303 & 2 \\
\hline 8 & 19 & 11.75 & 4.4328 & 2 \\
\hline 9 & 19 & 11.75 & 4.5250 & 2 \\
\hline 10 & 19 & 11.75 & 4.6079 & 2 \\
\hline
\end{tabular}

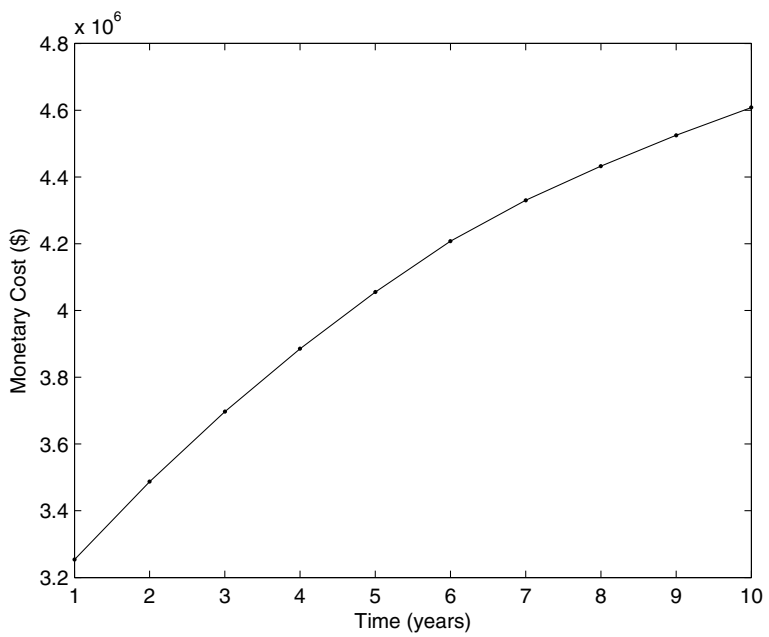

Fig. 7. Time horizon $\mathrm{x}$ Monetary cost.

The 10 year time horizon has been considered for doing another study too. In this case, the effect of the resolution parameter $r$ in the quality of the final solution (cost and substation position) has been analyzed. Resolutions of $4 \mathrm{~km}$, $2 \mathrm{~km}, 1 \mathrm{~km}, 0.5 \mathrm{~km}$ and $0.25 \mathrm{~km}$ have been considered. The results which have been observed are shown in Tab. III and Fig. 8.

From Tab. III, it is possible to note that the resolutions $r=4 k m$ and $r=2 \mathrm{~km}$ lead to the same results. When the resolution is increased from $r=2 \mathrm{~km}$ to $r=1 \mathrm{~km}$, then the monetary cost is reduced in about $0.50 \%$. Finally, when the resolution is set as $0.25 \mathrm{~km}$, the cost is reduced in $0.10 \%$ when compared to the $1.00 \mathrm{~km}$ and $0.50 \mathrm{~km}$. There is no significant improvement in cost for resolutions higher than $r=0.25 \mathrm{~km}$. These results suggest that it is not necessary to consider an excessively high resolution, since it does not lead to significant cost improvements.

\section{CONCLUSION}

In this paper, mechanisms for performing substation location jointly with distribution system topology design have been proposed. These mechanisms have been embedded inside the
TABLE III

NETWORK INFORMATION FOR EACH RESOLUTION.

\begin{tabular}{|c|c|c|c|c|}
\hline Resolution $(\mathrm{km})$ & $x_{s(i)}$ & $y_{s(i)}$ & $f(N, S) \times 10^{6}$ & Topology \\
\hline 4 & 20 & 12 & 4.6348 & 2 \\
\hline 2 & 20 & 12 & 4.6348 & 2 \\
\hline 1 & 19 & 12 & 4.6125 & 2 \\
\hline 0.5 & 19 & 12 & 4.6125 & 2 \\
\hline 0.25 & 19 & 11.75 & 4.6079 & 2 \\
\hline
\end{tabular}

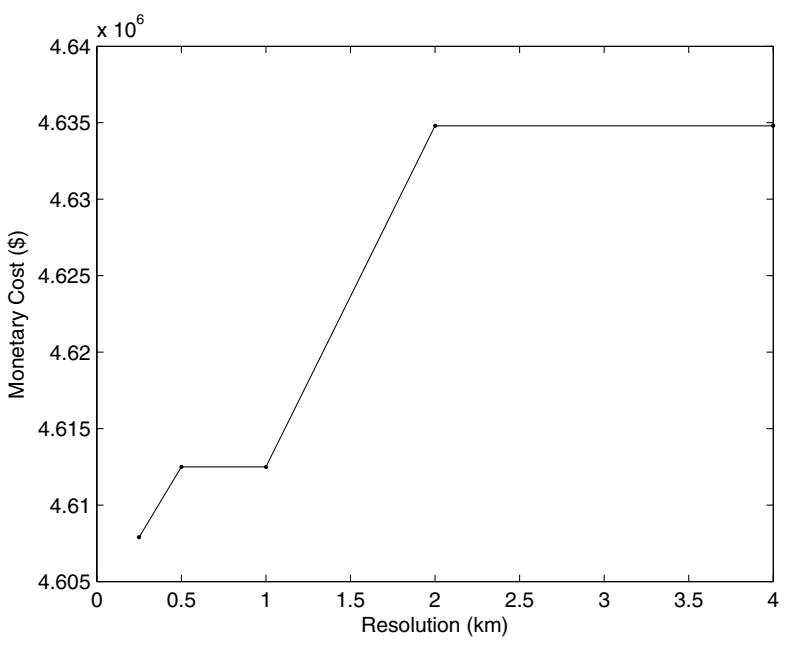

Fig. 8. Resolution x Monetary cost.

function evaluation block, what has made possible to develop the design tool without any adaptation on the original genetic algorithm.

With regard to results, the methodology proposed in this work has achieved satisfactory results for the electric energy distribution expansion problem, taking into account the minimization of the monetary cost of the achieved networks. Besides, the proposed algorithm is considerably faster than the previous ones, since it can reach a final solution (topology + substation positions) within a single GA run. The previous works required several runs of the GA and the BFGS algorithms for reaching a single solution [2]. Besides, the proposed mechanism is able to handle with disjoint candidate regions simultaneously.

As a future development, a multiobjective version of the proposed algorithm will be developed, in order to deal with reliability issues too.

\section{ACKNOWLEDGMENTS}

The authors acknowledge the support by the Brazilian agencies CAPES, CNPq and FAPEMIG.

\section{REFERENCES}

[1] S. Ćurčić, G. Strbac, and X. P. Zhang., "Effect of losses in design of distribution circuits," IEE Proceedings on Generation, Transmission and Distribution, vol. 148, no. 4, pp. 343-349, 2001.

[2] E. G. Carrano, R. H. C. Takahashi, E. P. Cardoso, R. R. Saldanha, and O. M. Neto, "Optimal substation location and energy distribution network design using a hybrid GA-BFGS algorithm," IEE Proceedings on Generation, Transmission and Distribution, vol. 152, no. 6, pp. 919926, 2005. 
[3] R. J. Vanderbei, Linear Programming: Foundations and Extensions. Springer, 2001.

[4] R. H. C. Takahashi, J. A. Vansconcelos, J. A. Ramirez, and L. Krahenbul, "A multiobjective methodology for evaluating genetic algorithms," in Proc. IEEE Conf. Computation of Electromagnetic Fields, Evian, France, 2002.

[5] D. E. Goldberg, Genetic Algorithms in Search, Optimization and Machine Learning. Addison-Wesley, 1989.

[6] E. G. Carrano, R. H. C. Takahashi, C. M. Fonseca, and O. M. Neto, "Bi-objective combined facility location and network design," Lecture Notes in Computer Science, vol. 4403, pp. 486-500, 2007.

[7] N. Srinivas and K. Deb, "Multi-objective optimization using nondominated sorting in genetic algorithms," Evolutionary Computation, vol. 2, no. 3, pp. 221-248, Fall 1994.

[8] K. Deb, A. Pratap, S. Agarwal, and T. Meyarivan, "A fast and elitist multiobjective genetic algorithm: NSGA II," IEEE Transactions on Evolutionary Computation, vol. 6, pp. 182-197, 2002.

[9] E. Zitzler and L. Thiele, "Multiobjective evolutionary algorithms: a comparative case study and the strength pareto approach," IEEE Transactions on Evolutionary Computation, vol. 3, no. 4, pp. 257-271, 1999.

[10] F. Mendoza, J. L. Bernal-Agustín, and J. A. Domínguez-Navarro, "NSGA and SPEA applied to multiobjective design of power distribution systems," IEEE Transactions on Power Systems, vol. 21, no. 4, pp. 1938$1945,2006$.

[11] E. G. Carrano, L. A. E. Soares, R. H. C. Takahashi, R. R. Saldanha, and O. M. Neto, "Electric distribution multiobjective network design using a problem-specific genetic algorithm," IEEE Transactions on Power Delivery, vol. 21, no. 2, pp. 995-1005, 2006.

[12] C. G. Taroco, E. G. Carrano, and O. M. Neto, "Robust design of power distribution systems using an enhanced multi-objective genetic algorithm," International Journal of Natural Computing Research, vol. 1, no. 2, pp. 92-112, 2010.

[13] J. Kennedy and R. C. Eberhart, "Particle swarm optimization," in IEEE International Conference on Neural Networks, Perth, WA, Australia, 1995.

[14] S. Ganguly, N. C. Sahoo, and D. Das, "Mono-and multi- objective planning of electrical distribution networks using particle swarm optimization," Applied Soft Computing, vol. 11, no. 2, pp. 2391-2405, 2011.

[15] "Genetic algorithms applied to the design of large power distribution systems," IEEE Transactions on Power Systems, vol. 13, no. 3, pp. 696702, 1998.

[16] H. L. Willis, H. Tram, M. V. Engel, and L. Finley, "Selecting and applying distribution optimization methods," IEEE Computer Aplications in Power, vol. 9, no. 1, pp. 12-17, 1996.

[17] E. G. Carrano, R. H. C. Takahashi, C. M. Fonseca, and O. M. Neto, "Non-linear network optimization - An embedding vector space approach," IEEE Transactions on Evolutionary Computation, vol. 14, pp. 206-226, 2010. 